\title{
Determinação da temperatura de neutralidade em salas de aula do ensino superior para as zonas bioclimáticas do estado de Mato Grosso
}

\author{
Determination of neutral temperature in higher \\ education classrooms in the bioclimatic zones of the state \\ of Mato Grosso, BR
}

\begin{abstract}
Karen Wrobel Straub
Erika Fernanda Toledo Borges Leão

Ernesto Kuchen

Marlon Leão

Resumo

$\mathbf{N}$

eutralidade térmica é a condição em que uma pessoa não prefira nem mais calor nem mais frio no ambiente ao seu redor, fator condicionante para atingir o conforto térmico. Tendo em vista que Mato Grosso apresenta significativas variações climáticas, evidenciouse a necessidade de estudos específicos, principalmente em instituições de ensino superior, visto que estas abrigam ocupantes por longos períodos e a aprendizagem está diretamente relacionada às condições de conforto dos estudantes. Este trabalho propôs determinar a temperatura de neutralidade em salas de aula de edificações do ensino superior, para as zonas bioclimáticas do Estado de Mato Grosso. A metodologia baseou-se na aplicação de questionários para verificação de variáveis pessoais e na medição de variáveis ambientais. A pesquisa foi realizada nos períodos seco e chuvoso, totalizando 64 medições com 1.151 questionários. Através de análise de regressão linear entre a temperatura operativa e o voto médio de sensação térmica emitido pelos ocupantes, ajustaram-se equações para a determinação da temperatura de neutralidade por zona bioclimática para as

Karen Wrobel Straub Universidade do Estado de Mato Grosso Sinop - MT - Brasil

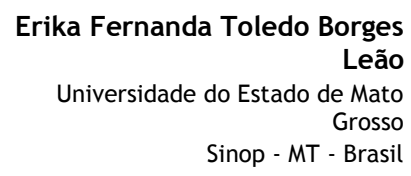

Ernesto Kuchen Universidad Nacional de San Juan Rivadavia - San Juan - Argentina

Marlon Leão Universidade do Estado de Mato
Grosso Sinop - MT - Brasil

Recebido em 26/03/16 Aceito em 20/06/16 estações de chuva e seca. A temperatura de neutralidade média encontrada para o Estado de Mato Grosso foi de $26,6^{\circ} \mathrm{C}$.

Palavras-chaves: Conforto térmico. Abordagem adaptativa. Pesquisa de campo.

\section{Abstract}

Thermal neutrality is a condition where a person does wish their environment to be neither cooler nor warmer, and it is a conditioning factor to achieve thermal comfort. The fact that the state of Mato Grosso presents significant climate variations through its territory highlights the need for specific studies, particularly in higher education institutions, as their occupants spend extended periods of time in classrooms and learning capacity of students is directly related to their comfort conditions. This paper evaluates neutral temperatures in classrooms in four bioclimatic zones of Mato Grosso state. The methodology is based on questionnaires for the verification of personal variables, and the measurement of climatic variables. The field research was conducted during the dry and rain seasons, totalling 64 cycles of measurement and 1151 questionnaires. Through an ordinary least square regression analysis between the operating temperature and the thermal sensation voting, the equations to determine the neutral temperature were adapted for each bioclimatic area for the rain and dry seasons. The average neutral temperature found for the state of Mato Grosso was $26.6^{\circ} \mathrm{C}$.

Keywords: Thermal comfort. Adaptive approach. Field research.
\end{abstract}

STRAUB, K. W.; LEÃO, E. F. B.; KUCHEN, E.; LEÃO, M. Determinação da temperatura de neutralidade em salas de aula do ensino superior para as zonas bioclimáticas do estado de Mato Grosso. Ambiente Construído, Porto Alegre, v. 17, n. 1, p. 97-109, jan./mar. 2017. 


\section{Introdução}

Os primeiros estudos realizados acerca da interferência das condições térmicas no rendimento de trabalhadores foram desenvolvidos em 1916, pela Comissão Americana de Ventilação. Inicialmente as pesquisas de conforto térmico eram realizadas em câmaras climatizadas com controle de todas as variáveis. Mais tarde surgiu a abordagem adaptativa, em que as pesquisas passaram a ser realizadas em ambientes reais.

O conforto térmico tem sido alvo de diversas pesquisas há muitos anos, com o objetivo principal de entender o seu funcionamento verificando quais as variáveis, os fatores e os índices podem interferir, e de que forma isso afeta a saúde e produtividade humana.

Segundo Ashrae (2005), conforto térmico é “a condição de espírito que manifesta a sua satisfação com o ambiente térmico e é avaliado por percepção subjetiva" (tradução nossa).

Essa abordagem é resultado de dois grandes grupos de pesquisas na área do conforto térmico, sendo a primeira realizada em câmaras climatizadas e a segunda proveniente de estudos em ambientes reais.

De acordo com Humphreys (1976), o interesse no estudo do modelo adaptativo pode ser identificado por duas razões principais: a primeira por ter se verificado resultados de estudos em câmaras climatizadas que divergem dos encontrados em ambientes reais; e a segunda pela constatação de que a população apresenta uma capacidade de se adaptar ao lugar em que vive e em detrimento disso aceita um intervalo de temperatura muito superior ao proposto pela abordagem estática.

Kuchen e Fisch (2009) afirmam que usuários adaptam-se a espaços com condições térmicas constantes definidas por um operador e que, portanto, esses antecedentes justificam a necessidade de elaborar um modelo de conforto variável em função de aspectos locais, próprios do edifício e dos usuários, corroborando a linha de pesquisa adaptativa.

De acordo com Kuchen et al. (2011), diversos trabalhos de campo mostram a combinação entre fatores ambientais e não ambientais e sua influência sobre o conforto térmico em ambientes reais de trabalho, e por isso vários autores, Hellwig e Bischof (2006), Boestra (2006), Raue et al. (2004), Nicol e Humphreys (2002), De Dear (2004) defendem que o emprego de normas como a ISO 7730 (INTERNATIONAL..., 2005), que se baseia no modelo analítico de Fanger, apresenta desvios que são próprios do método de obtenção desse modelo.

É importante ressaltar que embora a abordagem adaptativa tenha surgido posteriormente à estática e leve em consideração mais variáveis e fatores que possam afetar a sensação de conforto, o objetivo das duas abordagens é muito próximo: alcançar a condição de conforto térmico em ambientes reais.

Muitas pesquisas têm sido realizadas nesse sentido. Oseland (1994), em pesquisa realizada no Reino Unido, objetivou determinar a temperatura de neutralidade, comparando os votos médios preditos, utilizando o PMV de Fanger com as sensações relatadas por moradores de casas no período de verão e inverno, e verificou que em ambas as estações os limites apontados pelos moradores foram superiores aos calculados.

Humphreys (1976) realizou uma revisão dos parâmetros até então existentes, e concluiu que os índices de conforto variavam em relação à temperatura média da região onde cada estudo era desenvolvido, observando que a aclimatação afetava a temperatura de neutralidade, ao contrário do que mostravam os estudos de Fanger, que não sugeriam que a aclimatação afetasse os requisitos de conforto térmico.

Kuchen et al. (2011) desenvolveram uma ferramenta para avaliar o conforto térmico em espaços de trabalho de edifícios de escritório, através de uma pesquisa realizada em 30 edifícios na Alemanha, que consistiu em medições e questionários realizados no local. Com a correlação dos dados objetivos e subjetivos foi possível desenvolver um modelo estatístico para determinação da temperatura de neutralidade, que foi de $23,3{ }^{\circ} \mathrm{C}$, menor do que a encontrada com a utilização do modelo de Fanger.

Araújo (1996), em seus estudos de campo realizados em Natal, no Rio Grande do Norte, em ambientes escolares, verificou limites de temperaturas confortáveis entre $25,1^{\circ} \mathrm{C}$ e $28,1^{\circ} \mathrm{C}$.

Xavier (1999) realizou uma pesquisa no Estado de Santa Catarina, em ambiente escolar, com o objetivo de determinar, por meio de correlações estatísticas, índices e parâmetros de conforto térmico para aquela região, e verificou um intervalo de aceitabilidade térmica variando de 20 ${ }^{\circ} \mathrm{C}$ a $26{ }^{\circ} \mathrm{C}$. Segundo o autor, se comparado a outros trabalhos já realizados no país houve divergência nos resultados, e isso se dá principalmente em função da capacidade de adaptação das pessoas aos diferentes climas. 
Em pesquisa realizada no Mato Grosso do Sul por Andreasi e Lamberts (2006) com voluntários em atividades sedentárias em ambientes ventilados naturalmente, verificou-se um intervalo de aceitabilidade térmica que variou de $23{ }^{\circ} \mathrm{C}$ a 31 ${ }^{\circ} \mathrm{C}$.

Para Bueno (2006), a universidade pode e deve desempenhar um papel fundamental na consolidação do conceito e da prática de responsabilidade social, e, nesse sentindo, a pesquisa traz como proposta promover a interação da universidade com a sociedade, visto que através dos resultados obtidos torna-se possível a promoção de técnicas construtivas mais eficientes, e que venham atender as necessidades da população no que diz respeito ao conforto térmico.

Grzybowski (2004) afirma que a sala de aula de maneira geral e o que nela ocorre sempre foram assunto de muito interesse para administradores, pais e professores, e ainda ressalta que o ambiente físico de salas de aula pode ser um instrumento educacional poderoso, constituindo-se em um fator de influência indireta sobre o comportamento dos professores e alunos.

Em ambientes escolares é importante garantir a condição de conforto aos alunos, visto que estes estão expostos a diversos estímulos diferentes durante as aulas, e a insatisfação térmica com o ambiente pode interferir no processo de aprendizagem, tendo em vista que as reações fisiológicas dos alunos dependem diretamente das condições ambientais. Ressalta-se ainda que o excesso de calor dificulta a concentração e pode causar inquietação, que aliada à baixa umidade pode provocar sonolência e aumento de suor. Esses fatores podem causar estresse térmico e ao longo do tempo provocar doenças mais complexas. (DIAS, 2009; NOGUEIRA E NOGUEIRA, 2003; COUTINHO FILHO; SILVA; SILVA, 2007). Os fatos citados, aliados à importância do conforto térmico para ambientes, de uma forma geral justificam os estudos em instituições de ensino, principalmente de nível superior, visto que esses ambientes abrigam um grande número de ocupantes por longo período de tempo e que necessitam de um ambiente confortável, principalmente no que diz respeito à temperatura, para desenvolver as atividades eficientemente.

Tendo em vista que os índices de conforto variam em relação à temperatura média da região onde cada estudo é desenvolvido, observando ainda que a aclimatação, ou habilidade de adaptação, afeta a temperatura de neutralidade dos ambientes estudados, verifica-se a importância da determinação da temperatura de neutralidade para regiões específicas, visto que em um mesmo país existem regiões que apresentam condições climáticas muito diferentes e consequentemente habitantes com sensibilidade térmicas diferentes, justamente devido à condição de aclimatação do ser humano.

A ausência de zonas de conforto e temperatura de neutralidade conhecidas contribuem para edificações de baixo desempenho, no que diz respeito à condição térmica do ambiente, o que provoca gastos muitas vezes desnecessários com refrigeração. Com base nos pressupostos apresentados, o objetivo geral deste trabalho foi determinar a temperatura de neutralidade de salas de aula climatizadas de instituições do ensino superior para as zonas bioclimáticas do Estado de Mato Grosso.

\section{Área de estudo}

O Estado de Mato Grosso possui um extenso território com grandes variações climáticas e os mais diversos tipos de edificações, carecendo de zonas de conforto térmico e temperatura de neutralidade definidas. Está localizado na Região Centro-Oeste (IBGE, 2014), e apresenta em seu território dois climas diferentes, Equatorial e Tropical Brasil Central.

Entretanto, Maitelli (2005) afirma que devido à existência de muitas variações de temperatura, precipitação, vegetação, solo, topografia, latitude, altitude, além das alterações na superfície de fontes naturais ou de atividades humanas, e muitas vezes da insuficiência de dados meteorológicos, torna-se difícil uma classificação climática satisfatória.

De acordo com a autora, as classificações climáticas que se destacam são as apresentadas por Arthur Strahler e Wladimir Köppen. Segundo a classificação de Strahler, o Estado de Mato Grosso apresenta dois climas: Equatorial Quente e Úmido e Tropical Seco e Úmido.

De acordo com Tarifa (2011), o clima do Estado de Mato Grosso pode ser dividido em três unidades: I - Clima Equatorial Continental Úmido, com estação seca definida da Depressão SulAmazônica, II - Clima Subequatorial Continental Úmido, com estação seca definida do Planalto dos Parecis e III - Clima Tropical Continental Alternadamente Úmido e Seco das chapadas, planaltos e depressões de Mato Grosso, e todas elas ainda são divididas em subunidades, que dependem de suas semelhanças climáticas.

De acordo com a classificação das zonas bioclimáticas brasileiras da NBR 15220-3 (ABNT, 2005), o Estado de Mato Grosso apresenta 5 zonas bioclimáticas das 8 zonas presentes no país. 
Como Mato Grosso apresenta uma grande variedade de zonas bioclimáticas, e sabendo que o objetivo é determinar uma temperatura de neutralidade capaz de atender à condição de conforto para todo o Estado, torna-se necessária a verificação da temperatura de neutralidade em cada zona bioclimática, para posterior análise e generalização estadual.

A Tabela 1 traz a representação das cidades onde foram realizadas as pesquisas, bem como as zonas bioclimáticas às quais pertencem adotadas para esta pesquisa.

A classificação do zoneamento bioclimático do Estado de Mato Grosso pode ser observada na Figura 1.

\section{Método}

Para a determinação da temperatura de neutralidade em salas de aula de instituições de ensino superior, artificialmente climatizadas para as zonas bioclimáticas do Estado de Mato Grosso, a pesquisa foi realizada em duas etapas principais: pesquisas de campo e obtenção dos índices e tratamento estatístico dos dados. Os dados foram coletados no período chuvoso e no período de seca em cada uma das cidades.

Para o seu desenvolvimento foi utilizado o conceito Spot-Monitoring, que é fruto de uma pesquisa de doutorado de Kuchen (2008), realizado na Alemanha.

A escolha dos ambientes da pesquisa deu-se em função dos acordos de cooperação concedidos, e as sete instituições de ensino avaliadas apresentavam como estratégia de climatização a utilização de ar condicionado em todas as salas de aula.

Tabela 1 - Classificação bioclimática das cidades estudadas do Mato Grosso

\begin{tabular}{llc}
\hline UF & \multicolumn{1}{c}{ Cidade } & Zona bioclimática \\
\hline MT & Cáceres & 8 \\
MT & Cuiabá & 7 \\
MT & Primavera do Leste & 6 \\
MT & Sinop & 5 \\
\hline
\end{tabular}

Figura 1 - Zoneamento bioclimático segundo a NBR 15220-2003 para o Estado de Mato Grosso

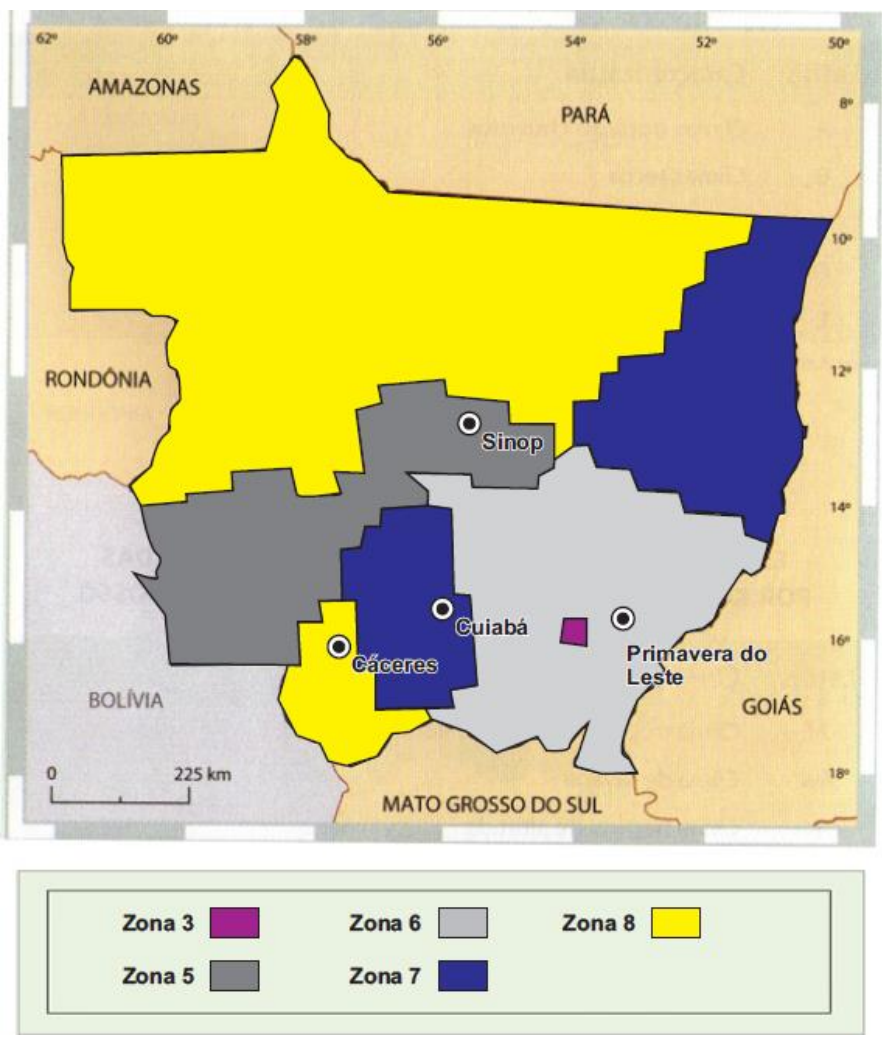

Fonte: adaptado de Sanches (2011).

100 Straub, K. W.; Leão, E. F. B.; Kuchen, E.; Leão, M. 
Caracterizaram-se os ambientes avaliados de acordo com a ISO 7726 (ISO, 1998), que considera um ambiente homogêneo do ponto de vista bioclimático aquele que em um dado momento a temperatura do ar, a radiação, a velocidade do ar e a umidade possam ser consideradas uniformes no espaço ao redor do ocupante, e é considerado como estacionário quando as variáveis físicas usadas para descrever o nível de exposição ao calor para a pessoa sejam praticamente independentes do tempo.

Para a coleta das variáveis ambientais, temperatura do ar, temperatura radiante média, umidade relativa e velocidade do ar, foi utilizado um conjunto de sensores, que, conectados a um datalogger, armazenavam as leituras realizadas para posterior transferência para o computador. A Figura 2 mostra o equipamento com as sondas utilizadas.

O armazenamento dos dados coletados foi realizado através do datalloger HD 32.1, instrumento que tem oito entradas para sondas módulo SICRAM, e os ajustes de calibração são memorizados internamente.

Para a realização da coleta dos dados subjetivos (opinião dos ocupantes sobre o ambiente térmico) e variáveis pessoais (idade, sexo, peso e vestimenta), fez-se uso de um questionário semelhante ao utilizado em outras pesquisas de avaliação de conforto térmico (KUCHEN et al., 2011). Este é específico para essa metodologia e foi desenvolvido em associação com o instituto de psicologia da Otto-von-Guericke-Universität de Magdeburg, na Alemanha, e adaptado para a realização desta pesquisa.

As sensações e preferências térmicas foram emitidas sobre a escala de sete pontos, na qual o usuário manifesta sua sensação térmica ao eleger os valores, que são -3 (muito frio), -2 (frio), -1 (um pouco frio), 0 (neutro $=$ conforto $),+1$ (um pouco calor), +2 (calor) e +3 (muito calor), com a possibilidade de escolher valores intermediários.

Para a coleta dos dados ambientais o equipamento ficou posicionado no centro das salas de aula para aclimatação anteriormente ao início das medições. As medições das variáveis ambientais seguiram as recomendações da norma ISO 7726 (ISO, 1998), com os sensores posicionados a $0,60 \mathrm{~m}$ de altura com relação ao solo.

As medições tiveram a duração de $5 \mathrm{~min}$, e o registro dos dados foi efetuado a cada $30 \mathrm{~s}$ e depois integrado para os $5 \mathrm{~min}$ da medição para determinar os valores médios. Simultaneamente, foram coletadas as variáveis pessoais e os parâmetros subjetivos através do questionário.

A taxa metabólica assumida única e constante durante as pesquisas foi igual a $70 \mathrm{~W} / \mathrm{m}^{2}$, conforme sugerido por outras pesquisas dessa linha (XAVIER, 1999).

As coletas aconteceram nos períodos matutino, vespertino e noturno, em todas as zonas bioclimáticas estudadas, durante as estações chuvosa (fevereiro e março) e seca (junho, setembro e outubro).

Pelos votos de sensação térmica dos ocupantes, manifestados no questionário, no momento da medição foi calculada a média dos votos por sala de aula, obtendo-se dessa forma o voto médio de sensação térmica.

Visando o estabelecimento das relações de dependência entre as variáveis, bem como a definição das equações para a determinação das temperaturas de neutralidade, para salas de aula do ensino superior em edifícios condicionados artificialmente do Estado de Mato Grosso, foram realizadas análises de regressão de mínimos quadrados.

Figura 2 - Datalogger e sensores utilizados
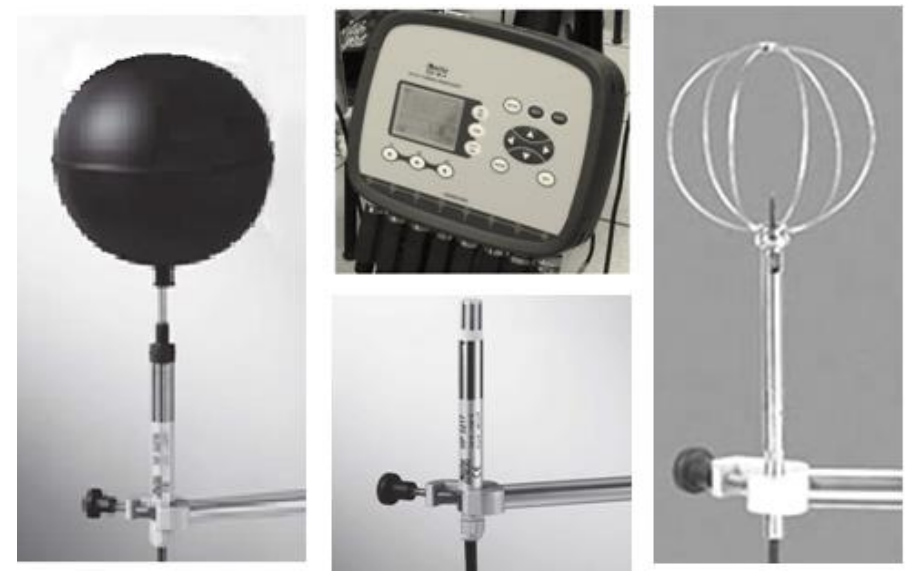
Através das regressões determinou-se uma equação para cada zona bioclimática do Estado de Mato Grosso, e para cada estação, chuva e seca.

Verifica-se que a interseção da reta, ajustada pela regressão, com os valores de +1 e -1 , permite encontrar um intervalo de temperatura operativa limitado entre os significados um pouco frio e um pouco quente. Esse intervalo evidencia a amplitude da aceitabilidade térmica dos ocupantes.

Para a determinação dos valores de neutralidade térmica por zona bioclimática do Estado de Mato Grosso, inseriu-se às equações de regressão determinadas para o voto de sensação térmico igual a "zero", sensação de conforto, dessa forma encontra-se uma temperatura operativa que coincide com a temperatura de neutralidade.

Para a validação de todas as regressões foram realizadas análises de variância, bem como os testes de normalidade de Kolmogorov-Smirnov e Shapiro-Wilk dos resíduos.
Para a realização da pesquisa foram consultados 1.151 alunos (votos) nos períodos de chuva e seca, sobre a sensação térmica, com um total de 64 medições nas quatro zonas bioclimáticas do Estado de Mato Grosso.

\section{Resultados}

Das 64 medições, realizadas nos períodos de chuva e seca, obtiveram-se 1.151 questionários válidos, valor este que se aproxima consideravelmente de outras pesquisas realizadas nessa linha, como a de Fanger (1970), com 1.300 questionários, a de Xavier (1999), com 1.415, e a de Kuchen (2008), com 1.100 questionários.

Dentre os 1.151 questionários respondidos $55,24 \%$ foram respondidos por mulheres e $44,76 \%$ por homens. A Tabela 2 traz as médias das principais variáveis pessoais e ambientais para cada zona bioclimática nas estações chuvosa e seca.

Tabela 2 - Média e desvio padrão das variáveis coletadas

\begin{tabular}{|c|c|c|c|c|c|c|c|c|c|c|c|}
\hline \multicolumn{12}{|c|}{ Período de chuva } \\
\hline & Zona & $\begin{array}{c}\mathbf{R} \\
\text { (clo) }\end{array}$ & $\begin{array}{c}\text { Ta } \\
\left({ }^{\circ} \mathrm{C}\right) \\
\end{array}$ & $\begin{array}{c}\mathbf{V a} \\
(\mathbf{m} / \mathbf{s})\end{array}$ & $\begin{array}{l}\text { Tmr } \\
\left({ }^{0} \mathrm{C}\right)\end{array}$ & $\begin{array}{l}\text { UR } \\
(\%)\end{array}$ & PMV & $\begin{array}{l}\text { PPD } \\
(\%)\end{array}$ & $\begin{array}{l}\text { Top } \\
\left({ }^{\circ} \mathrm{C}\right) \\
\end{array}$ & $\mathbf{S}$ & $\begin{array}{c}\text { I } \\
(\%)\end{array}$ \\
\hline \multirow[b]{2}{*}{8} & Média & 0,41 & 26,5 & 0,13 & 26,8 & 65,13 & 0,05 & 9,32 & 26,6 & $-0,10$ & 25,42 \\
\hline & $\begin{array}{l}\text { Desvio } \\
\text { padrão }\end{array}$ & 0,03 & 1,16 & 0,08 & 0,76 & 6,32 & 0,44 & 3,87 & 0,92 & 0,51 & 8,76 \\
\hline \multirow[b]{2}{*}{7} & Média & 0,48 & 24,7 & 0,10 & 25,6 & 44,32 & $-0,22$ & 9,15 & 25,2 & $-0,41$ & 29,47 \\
\hline & $\begin{array}{l}\text { Desvio } \\
\text { padrão }\end{array}$ & 0,16 & 7,93 & 0,05 & 8,29 & 14,09 & 0,42 & 4,44 & 8,12 & 0,59 & 17,20 \\
\hline \multirow[b]{2}{*}{6} & Média & 0,47 & 25,7 & 0,07 & 26,7 & 56,32 & 0,14 & 14,46 & 26,2 & 0,21 & 32,35 \\
\hline & $\begin{array}{l}\text { Desvio } \\
\text { padrão }\end{array}$ & 0,11 & 6,27 & 0,02 & 6,53 & 17,97 & 0,65 & 11,79 & 6,39 & 0,71 & 20,81 \\
\hline \multirow[b]{2}{*}{5} & Média & 0,46 & 25,5 & 0,10 & 26,5 & 53,03 & $-0,02$ & 6,87 & 25,9 & $-0,03$ & 24,89 \\
\hline & $\begin{array}{l}\text { Desvio } \\
\text { padrão }\end{array}$ & 0,12 & 6,44 & 0,06 & 6,68 & 12,21 & 0,33 & 3,12 & 6,55 & 0,30 & 5,95 \\
\hline \multicolumn{12}{|c|}{ Período de seca } \\
\hline & Zona & $\begin{array}{c}\mathbf{R} \\
\text { (clo) } \\
\end{array}$ & $\begin{array}{r}\text { Ta } \\
\left({ }^{\circ} \mathrm{C}\right) \\
\end{array}$ & $\begin{array}{c}\mathbf{V a} \\
(\mathbf{m} / \mathbf{s}) \\
\end{array}$ & $\begin{array}{l}\text { Tmr } \\
\left({ }^{0} \mathrm{C}\right) \\
\end{array}$ & $\begin{array}{l}\text { UR } \\
(\%) \\
\end{array}$ & PMV & $\begin{array}{r}\text { PPD } \\
(\%) \\
\end{array}$ & $\begin{array}{l}\text { Top } \\
\left({ }^{\circ} \mathrm{C}\right) \\
\end{array}$ & $\mathbf{S}$ & $\begin{array}{c}\text { I } \\
(\%) \\
\end{array}$ \\
\hline \multirow[b]{2}{*}{8} & Média & 0,40 & 27,2 & 0,37 & 28,6 & 47,45 & 0,27 & 17,69 & 27,8 & 0,56 & 38,55 \\
\hline & $\begin{array}{l}\text { Desvio } \\
\text { padrão }\end{array}$ & 0,08 & 1,45 & 0,41 & 1,30 & 5,88 & 0,76 & 11,76 & 1,20 & 0,57 & 20,81 \\
\hline \multirow[b]{2}{*}{7} & Média & 0,44 & 26,4 & 0,16 & 27,5 & 54,17 & 0,30 & 12,16 & 26,8 & $-0,27$ & 29,76 \\
\hline & $\begin{array}{l}\text { Desvio } \\
\text { padrão }\end{array}$ & 0,03 & 1,17 & 0,10 & 1,16 & 4,72 & 0,49 & 7,00 & 0,90 & 0,42 & 11,04 \\
\hline \multirow[b]{2}{*}{6} & Média & 0,48 & 26,6 & 0,08 & 28,1 & 49,14 & 0,66 & 26,89 & 27,3 & $-0,13$ & 21,40 \\
\hline & $\begin{array}{l}\text { Desvio } \\
\text { padrão }\end{array}$ & 0,16 & 9,24 & 0,08 & 9,98 & 18,81 & 0,79 & 20,75 & 9,66 & 0,36 & 12,86 \\
\hline \multirow[b]{2}{*}{5} & Média & 0,46 & 24,3 & 0,12 & 25,3 & 50,90 & $-0,30$ & 24,53 & 24,8 & $-0,18$ & 39,85 \\
\hline & $\begin{array}{l}\text { Desvio } \\
\text { padrão }\end{array}$ & 0,04 & 2,82 & 0,07 & 2,42 & 10,63 & 0,97 & 15,65 & 2,62 & 0,75 & 8,52 \\
\hline
\end{tabular}


A nomenclatura utilizada nas tabelas acima possui a seguinte representação:

$\mathrm{Z}$ : zona bioclimática onde foi realizada a pesquisa (Zona 8 - Cáceres; Zona 7 - Cuiabá; Zona 6 Primavera do Leste e; Zona 5 - Sinop);

$\mathrm{R}$ : isolamento térmico médio das vestimentas utilizadas pelos alunos durante a medição, em clo;

Tmr: temperatura média radiante, $\mathrm{em}{ }^{\circ} \mathrm{C}$;

PMV: voto médio predito, proposta por Fanger (1970), adimensional;

PPD: porcentagem de pessoas insatisfeitas, proposta por Fanger (1970);

Top: temperatura operativa média, expressa em ${ }^{\circ} \mathrm{C}$;

$\mathrm{S}$ : voto médio de sensação térmica, determinado através da média aritmética de todos os votos emitidos pelos ocupantes, por meio dos questionários, durante as medições de cada sala de aula, adimensional; e

I: porcentagem de insatisfeitos verificados na medição, como todos aqueles que votaram $-3,-2$, +2 e +3 , mais $50 \%$ dos que votaram +1 e -1 por meio do questionário, conforme realizado por Xavier (1999).

A correlação entre os votos médios de sensação térmica reais e a porcentagem de insatisfeitos reais (obtidos por meio do questionário) pode ser verificada na Figura 3, e não é tão estreita e acentuada quanto à apregoada pelo modelo de Fanger (1970), pois para este estudo de campo encontrou-se $\mathrm{R}^{2}=0,71$. Verifica-se ainda uma maior dispersão no percentual de insatisfeitos reais para as sensações próximas a zero, situação em que o ocupante encontra-se confortável.
Sendo que:

I: porcentagem de insatisfeitos reais verificados na medição, em \%; e

$\mathrm{S}$ : voto médio de sensação térmica, adimensional.

Um aspecto que pode justificar a maior variação quando o ambiente térmico se aproxima de uma situação de conforto é o tipo de climatização. Segundo Kuchen et al. (2011), pessoas que permanecem por longos períodos em espaços com climatização artificial experimentam mínimas variações térmicas, e, por isso, são mais sensíveis a mudanças de temperatura, o que nos permite concluir que esses ocupantes são muito mais exigentes na determinação de uma situação de conforto, evidenciando, dessa forma, a dispersão verificada próximo a zero.

Ao relacionar o PMV de Fanger (1970) com os votos de sensação térmica reais, Figura 4, verificase que apenas $47,92 \%\left(\mathrm{R}^{2}=0,4792\right)$ das variações das sensações reais coincidem com o modelo do PMV, enquanto 52,08\% dessas variações não coincidem com tal modelo.

A Equação 2 que representa a regressão da Figura 4:

$\mathrm{PMV}=0,61 S-0,24$

Eq. 2

Sendo que:

PMV: voto médio predito, proposto por Fanger (1970), adimensional; e

$\mathrm{S}$ : voto médio de sensação térmica, adimensional.

A Figura 5 traz a regressão linear entre o voto médio de sensação térmica e a temperatura operativa para a zona 8 , avaliação realizada na cidade de Cáceres, nos períodos de chuva e seca.

A Equação 1 representa a curva da Figura 3:

$I=26,08 S^{2}-1,10 S+21,44 \quad$ Eq. 1

Figura 3 - Correlação entre sensação real e porcentagem de insatisfeitos real

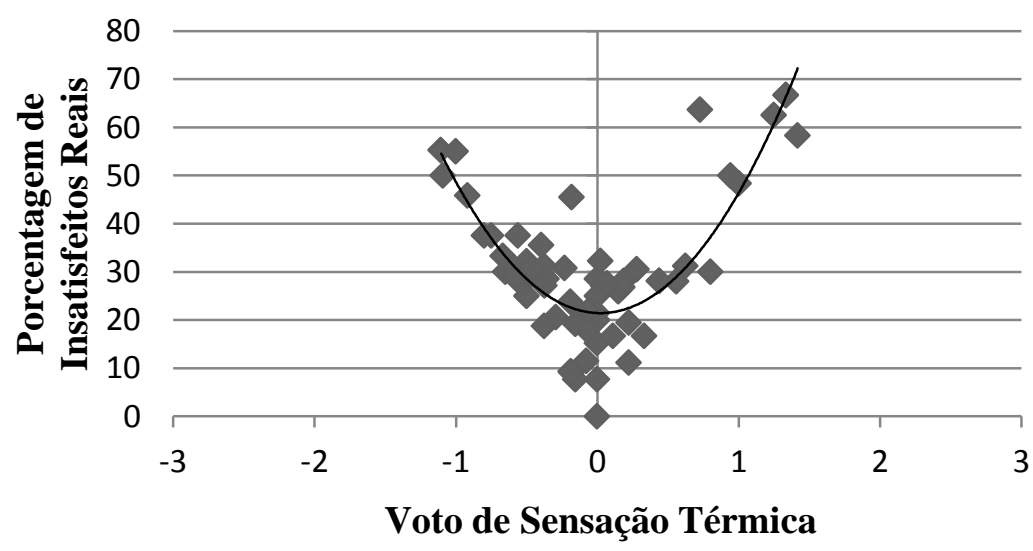


Figura 4 - Correlação entre PMV e sensações reais obtidas através dos questionários

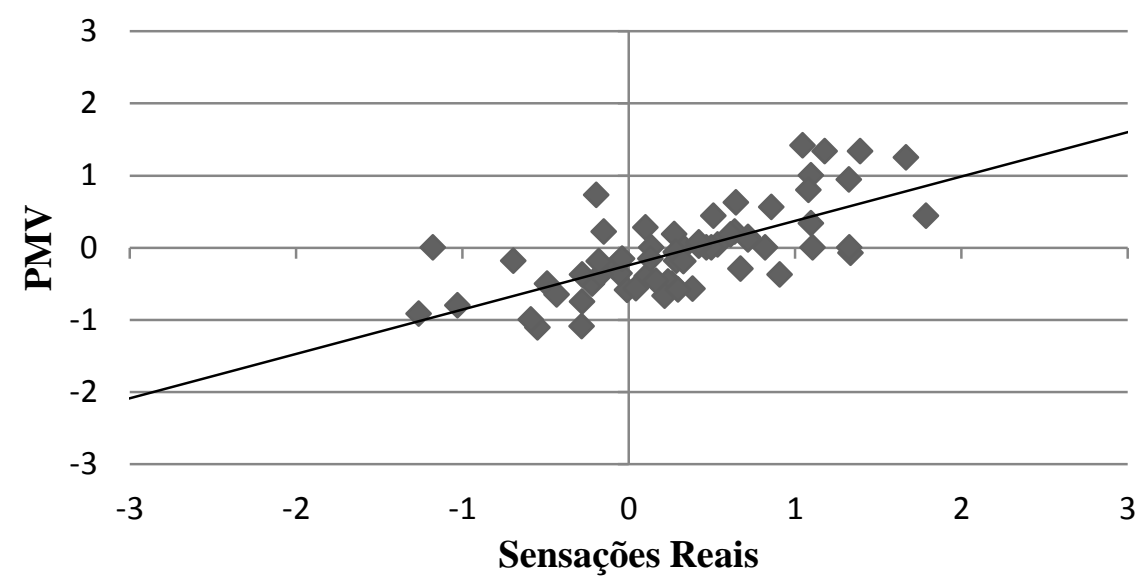

Figura 5 - Regressão entre voto de sensação térmica e temperatura operativa para a zona bioclimática 8

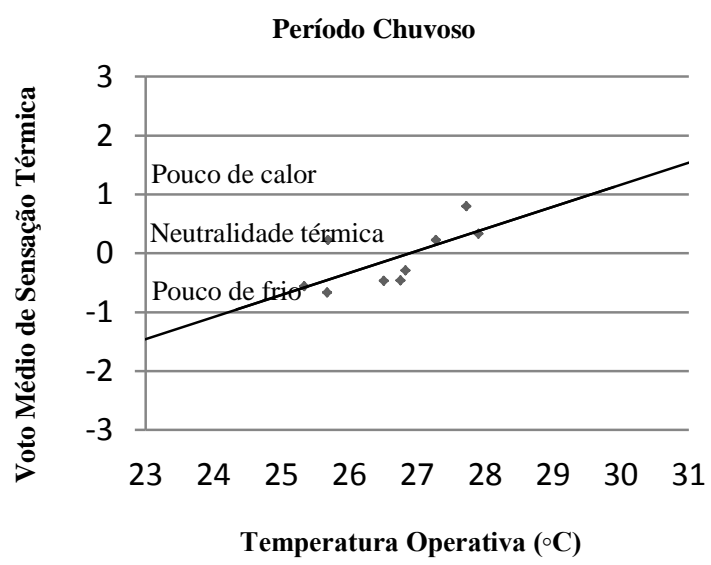

As Equações 3 e 4 representam as regressões para a zona 8 nas estações de chuva e seca, respectivamente.

$S=0,37 T_{o p}-10,07$

Eq. 3

$\mathrm{S}=0,36 T_{o p}-9,68$

Eq. 4

Sendo que:

$\mathrm{S}$ : voto médio de sensação térmica, adimensional; e

Top: temperatura operativa média, expressa em ${ }^{\circ} \mathrm{C}$.

A Tabela 3 apresenta os valores do intervalo de aceitabilidade térmica, a temperatura de neutralidade, bem como o coeficiente de correlação de Pearson.

A Figura 6 traz a regressão linear entre o voto de sensação térmica e a temperatura operativa para a

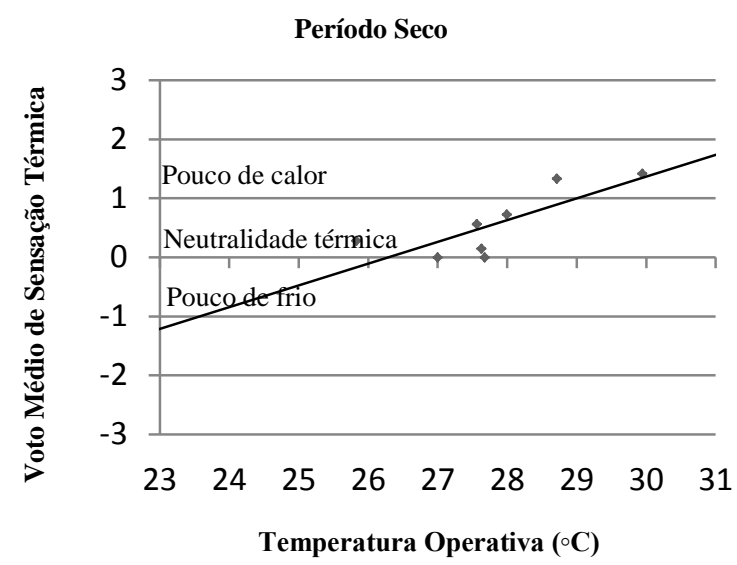

zona 7, avaliação realizada na cidade de Cuiabá, nos períodos de chuva e seca.

As Equações 5 e 6 representam as regressões para a zona 7 nas estações de chuva e seca, respectivamente. As demais informações podem ser verificadas na Tabela 4.

$S=0,41 T_{o p}-10,96$

Eq. 5

$S=0,35 T_{o p}-9,84$

Eq. 6

Sendo que:

$\mathrm{S}$ : voto médio de sensação térmica, adimensional; $\mathrm{e}$

Top: temperatura operativa média, expressa em ${ }^{\circ} \mathrm{C}$.

A Figura 7 traz a regressão linear entre o voto de sensação térmica e a temperatura operativa para a zona 6, avaliação realizada na cidade de Primavera do Leste, nos períodos de chuva e seca. 
Tabela 3 - Caracterização dos resultados para a zona bioclimática 8

\begin{tabular}{c|c|c|c|c|c|c}
\hline Período & $\begin{array}{c}\text { Salas de } \\
\text { aula }\end{array}$ & Questionários & $\mathbf{R}^{\mathbf{2}}$ & $\begin{array}{c}\text { Coeficiente } \\
(\mathbf{a})\end{array}$ & $\begin{array}{c}\text { Intervalo de } \\
\text { aceitabilidade }\end{array}$ & $\begin{array}{c}\text { Temperatura } \\
\text { de neutralidade }\end{array}$ \\
\hline Chuvoso & 9 & 147 & 0,46 & 0,37 & $24,2^{\circ} \mathrm{C}$ a $29,6^{\circ} \mathrm{C}$ & $26,9^{\circ} \mathrm{C}$ \\
Seco & 8 & 117 & 0,61 & 0,36 & $23,6^{\circ} \mathrm{C} \mathrm{a} 28,9^{\circ} \mathrm{C}$ & $26,3^{\circ} \mathrm{C}$ \\
\hline
\end{tabular}

Figura 6 - Regressão entre voto de sensação térmica e temperatura operativa para a zona bioclimática 7

Período Chuvoso

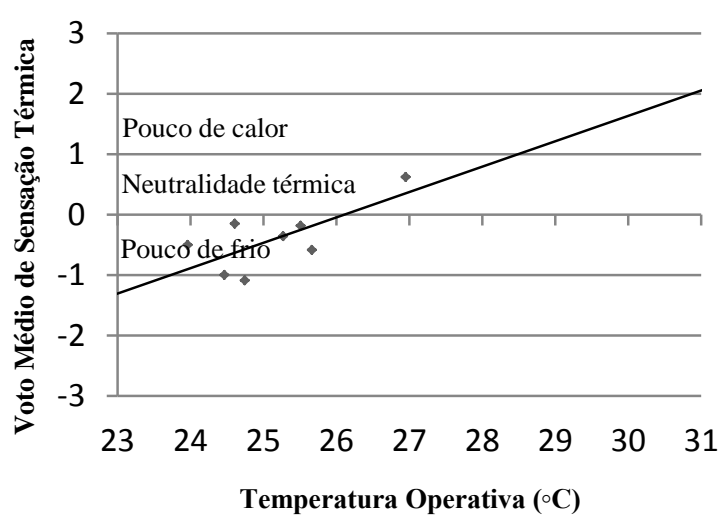

Período Seco

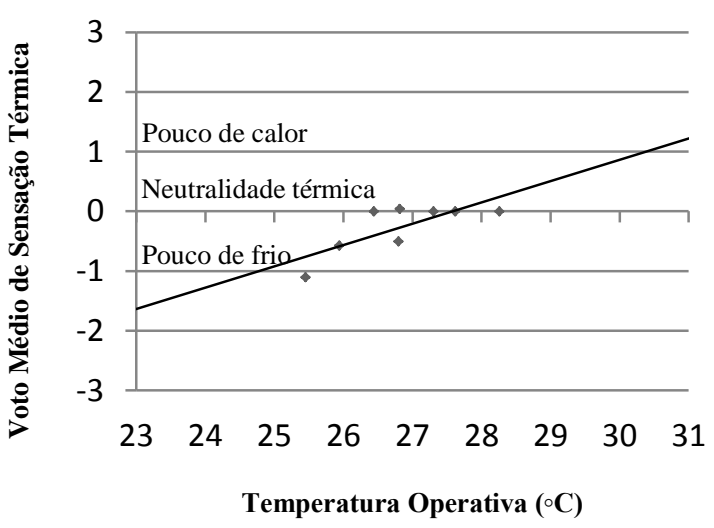

Tabela 4 - Caracterização dos resultados para a zona bioclimática 7

\begin{tabular}{ccccccc}
\hline Período & $\begin{array}{c}\text { Salas de } \\
\text { aula }\end{array}$ & Questionários & $\mathbf{R}^{\mathbf{2}}$ & Coeficiente (a) & $\begin{array}{c}\text { Intervalo de } \\
\text { aceitabilidade }\end{array}$ & $\begin{array}{c}\text { Temperatura } \\
\text { de neutralidade }\end{array}$ \\
\hline Chuvoso & 8 & 110 & 0,51 & 0,41 & $23,7{ }^{\circ} \mathrm{C}$ a $28,5^{\circ} \mathrm{C}$ & $26,1^{\circ} \mathrm{C}$ \\
Seco & 8 & 126 & 0,59 & 0,35 & $24,8^{\circ} \mathrm{C}$ a $30,4{ }^{\circ} \mathrm{C}$ & $27,6^{\circ} \mathrm{C}$ \\
\hline
\end{tabular}

Figura 7 - Regressão entre voto de sensação térmica e temperatura operativa para a zona bioclimática 6

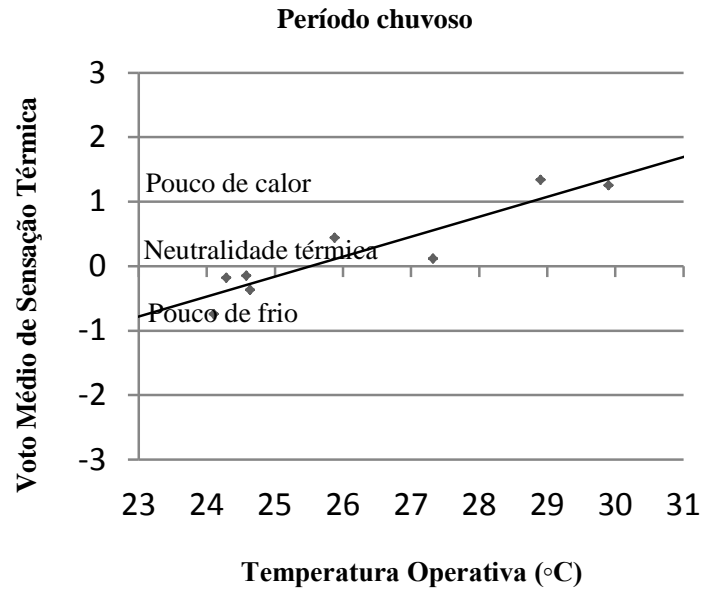

As Equações 7 e 8 representam as regressões para a zona 6 nas estações de chuva e seca, respectivamente. As demais informações podem ser verificadas na Tabela 5 .

$$
\begin{aligned}
& S=0,30 T_{o p}-7,89 \\
& S=0,08 T_{o p}-2,35
\end{aligned}
$$

Eq. 7

Eq. 8

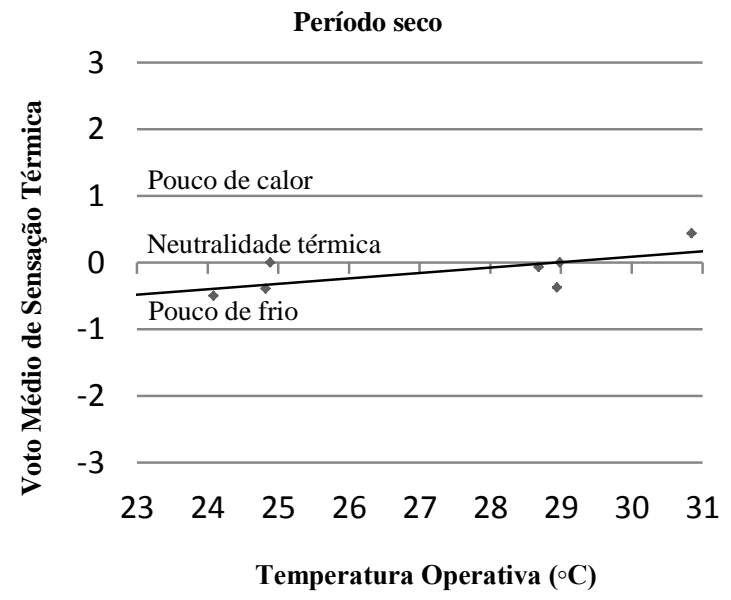

Sendo que:

S: voto médio de sensação térmica, adimensional;

Top: temperatura operativa média, expressa em ${ }^{\circ} \mathrm{C}$.
A Figura 8 traz a regressão linear entre o voto de sensação térmica e a temperatura operativa para a 
zona 5, avaliação realizada na cidade de Sinop, nos períodos de chuva e seca. As Equações 9 e 10 representam as regressões para a zona 6 nas estações de chuva e seca, respectivamente. As demais informações podem ser verificadas na Tabela 6.

$S=0,28 T_{o p}-7,44$

$S=0,27 T_{o p}-6,95$

Eq. 10

Sendo que:

$\mathrm{S}$ : voto médio de sensação térmica, adimensional; e

Top: temperatura operativa média, expressa em ${ }^{\circ} \mathrm{C}$.

As análises de variância demonstraram que as equações de regressão determinadas eram significativas, bem como os testes de KolmogorovSmirnov e Shapiro-Wilk dos resíduos evidenciaram a normalidade dos resíduos e, portanto, validaram as regressões lineares realizadas.

A Tabela 7 traz o resumo dos valores determinados de temperatura de neutralidade para cada zona bioclimática estudada, bem como o intervalo de aceitabilidade térmica e o valor do coeficiente "a" das equações de regressão. É importante destacar que todas as análises de regressão foram realizadas para um intervalo de confiança de $95 \%$.

Verifica-se que os valores do coeficiente "a" variaram bastante, tanto no que diz respeito às zonas quanto às estações. Tendo em vista que este coeficiente está relacionado à habilidade de adaptação dos ocupantes dos ambientes estudados, optou-se pela determinação da temperatura de neutralidade por zona bioclimática, fornecendo, dessa forma, subsídios mais específicos para cada região.

\section{Considerações finais}

A pesquisa foi realizada nas duas estações, seca e chuvosa, entre os anos de 2014 e 2015 e nas quatro zonas bioclimáticas do estado $(5,6,7$ e 8$)$. Foram medidas todas as variáveis ambientais de interesse nas salas de aula climatizadas artificialmente, bem como foram levantadas as variáveis pessoais dos ocupantes e suas sensações térmicas, o que possibilitou a determinação das temperaturas de neutralidade para salas de aula.

Tabela 5 - Caracterização dos resultados para a zona bioclimática 6

\begin{tabular}{ccccccc}
\hline Período & $\begin{array}{c}\text { Salas } \\
\text { de aula }\end{array}$ & Questionários & $\mathbf{R}^{\mathbf{2}}$ & Coeficiente (a) & $\begin{array}{c}\text { Intervalo de } \\
\text { aceitabilidade }\end{array}$ & $\begin{array}{c}\text { Temperatura } \\
\text { de neutralidade }\end{array}$ \\
\hline Chuvoso & 8 & 10 & 0,85 & 0,30 & $22,3{ }^{\circ} \mathrm{C} \mathrm{a} 28,8^{\circ} \mathrm{C}$ & $25,5^{\circ} \mathrm{C}$ \\
Seco & 7 & 142 & 0,44 & 0,08 & $16,6^{\circ} \mathrm{C} \mathrm{a} 41,2{ }^{\circ} \mathrm{C}$ & $28,9^{\circ} \mathrm{C}$ \\
\hline
\end{tabular}

Figura 8 - Regressão entre voto de sensação térmica e temperatura operativa para a zona bioclimática 5
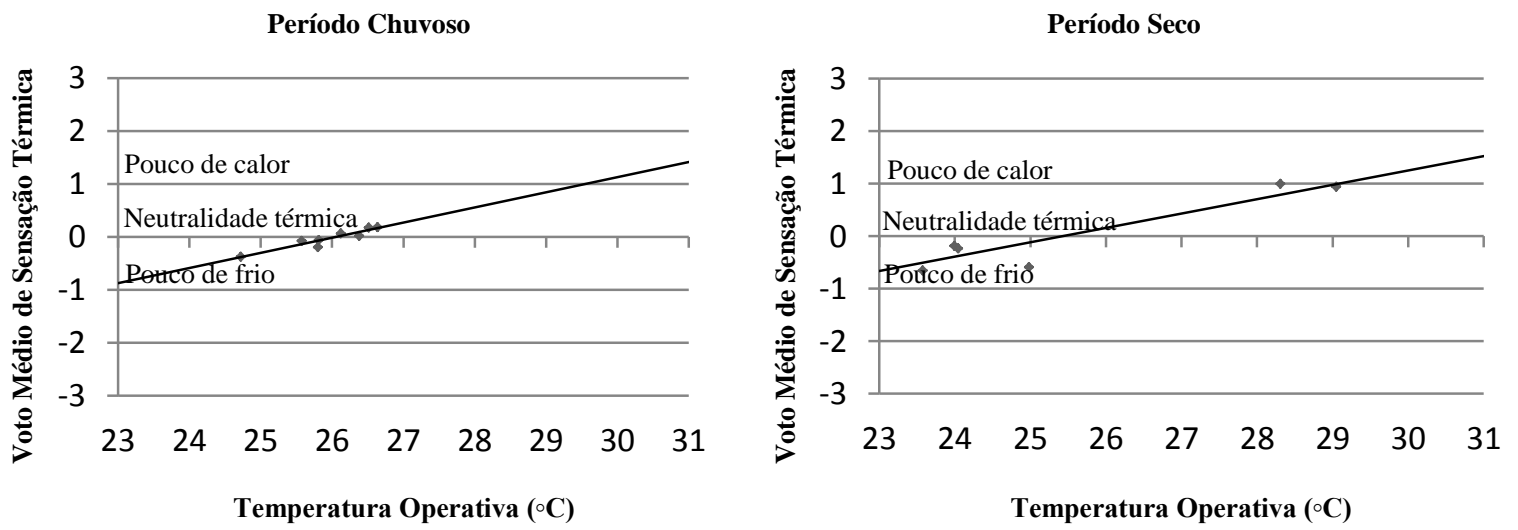

Tabela 6 - Caracterização dos resultados para a zona bioclimática 6

\begin{tabular}{ccccccc}
\hline Período & $\begin{array}{c}\text { Salas } \\
\text { de aula }\end{array}$ & Questionários & $\mathbf{R}^{\mathbf{2}}$ & Coeficiente (a) & $\begin{array}{c}\text { Intervalo de } \\
\text { aceitabilidade }\end{array}$ & $\begin{array}{c}\text { Temperatura } \\
\text { de neutralidade }\end{array}$ \\
\hline Chuvoso & 8 & 235 & 0,88 & 0,28 & $22,6^{\circ} \mathrm{C}$ a $29,6^{\circ} \mathrm{C}$ & $26,1^{\circ} \mathrm{C}$ \\
Seco & 8 & 174 & 0,90 & 0,27 & $21,8^{\circ} \mathrm{C} \mathrm{a} 29,1^{\circ} \mathrm{C}$ & $25,4^{\circ} \mathrm{C}$ \\
\hline
\end{tabular}

106 Straub, K. W.; Leão, E. F. B.; Kuchen, E.; Leão, M. 
Ambiente Construído, Porto Alegre, v. 17, n. 1, p. 97-109, jan./mar. 2017.

Tabela 7 - Temperatura de neutralidade por zona bioclimática do Estado de Mato Grosso

\begin{tabular}{ccccc}
\hline Estação & Zona & Coeficiente "a" & $\begin{array}{c}\text { Intervalo de aceitabilidade } \\
\left({ }^{\circ} \mathbf{C}\right)\end{array}$ & $\begin{array}{c}\text { Temperatura de neutralidade } \\
\left({ }^{\circ} \mathbf{C}\right)\end{array}$ \\
\hline \multirow{3}{*}{ Chuva } & 8 & 0,37 & $24,2-29,6$ & 26,9 \\
& 7 & 0,41 & $23,7-28,5$ & 26,1 \\
& 6 & 0,30 & $22,3-28,8$ & 25,5 \\
& 5 & 0,28 & $22,6-29,6$ & 26,1 \\
\hline \multirow{3}{*}{ Seca } & 8 & 0,36 & $23,6-28,9$ & 26,3 \\
& 7 & 0,35 & $24,8-30,4$ & 27,6 \\
& 6 & 0,08 & $16,6-41,2$ & 28,9 \\
\hline
\end{tabular}

A Tabela 7 apresentou resumidamente os resultados obtidos, e a temperatura de neutralidade média encontrada para o Estado de Mato Grosso, considerando as medições realizadas nas duas estações (chuva e seca), foi de $26,6^{\circ} \mathrm{C}$.

Os estudos relacionados ao conforto térmico, embora não muito recentes, têm tido forte destaque nos últimos anos principalmente quando associados à eficiência energética das edificações. As duas grandes linhas de pesquisas têm sido cada vez mais retroalimentadas. Embora as pesquisas de campo e pesquisas em câmaras climatizadas sejam bastante divergentes em sua metodologia e resultados obtidos, ambas têm os mesmo objetivos: o avanço do conhecimento acerca do conforto térmico e a determinação de formas para proporcioná-lo aos ocupantes das edificações.

Destaca-se aqui a importância da realização de pesquisas de campo, em que as condições e sensações reais são levantadas, visto que neste trabalho encontraram-se variações entre as sensações reais levantadas na pesquisa e as preconizadas pelo modelo analítico do PMV.

Os resultados obtidos, por meio do modelo adaptativo, corroboram com outros estudos realizados por Nicol e Humphreys (2002), Xavier (1999), Kuchen (2008) e outros, já que demonstram que a interpretação de conforto térmico é diferenciada para distintas populações, o que pode se tornar ainda mais evidentes quando comparados os resultados encontrados para cada zona bioclimática. A diferença encontrada justifica-se justamente pela característica de aclimatação do ser humano. Verificam-se ainda intervalos de aceitabilidade térmica condizentes com as condições climáticas de cada região.

O coeficiente "a" é um indicador da adaptação térmica ao intervalo de temperatura proporcionado pelo sistema de climatização. Valores mais baixos do coeficiente "a" correspondem a maior grau de adaptação térmica dos usuários ao ambiente construído. A capacidade de adaptação dos usuários deve ser considerada como um potencial de economia no consumo de energia em edifícios. Os próximos estudos devem considerar o comportamento deste indicador ao longo do tempo com o objetivo de desenvolver previsões.

Destaca-se, portanto, que através das equações de regressão foi possível determinar a temperatura de neutralidade para todas as zonas bioclimáticas do Estado de Mato Grosso no período chuvoso e seco, bem como o intervalo térmico de aceitabilidade.

Entretanto, ressalta-se que elas podem ser aplicadas apenas para grupos de pessoas que estejam desenvolvendo a mesma atividade e com entorno ambiental muito semelhante ao realizado neste estudo.

Ainda assim a pesquisa traz importantes inovações no que diz respeito às contribuições para alcançar o conforto térmico em espaços reais de trabalho e alavancar o desenvolvimento de estratégias que contribuam para a eficiência energética das edificações de ensino superior no Estado de Mato Grosso.

\section{Referências}

\section{AMERICAN SOCIETY OF HEATING, REFRIGERATING AND AIR CONDITIONING ENGINEERS. Energy Estimating Methods. In:} ASHRAE Handbook: fundamentals. New York: 2005.

ANDREASI, W. A.; LAMBERTS, R. Thermal Comfort in Buildings Located in Regions of Hot and Humid Climate of Brazil. 2006. Disponível em: < http://pgquimica.sites.ufms.br/wpcontent/blogs.dir/117/files/2015/01/Thermalcomfort-in-buildings-located-in-regions-of-hotand-humid-climate-of-Brazil-NCEUB-2009.pdf>. Acesso em: 02 set. 2016.

\section{ARAÚJO, V. M. D. Parâmetros de Conforto Térmico Para Usuários de Edificações Escolares no Litoral Nordestino Brasileiro.. São Paulo, 1996. Tese (Doutorado em Engenharia Civil) - Faculdade de Arquitetura e Urbanismo, Universidade de São Paulo, São Paulo,1996.}


ASSOCIAÇÃO BRASILEIRA DE NORMA TÉCNICAS. NBR 15220-3: desempenho térmico de edificações: parte 3: zoneamento bioclimático brasileiro e diretrizes construtivas para habitações unifamiliares de interesse social. Rio de Janeiro, 2005.

BOESTRA, A. C. The Adaptive Thermal Comfort Criterion in the New EPBD IEQ Standard. In: BBA Indoor Environmental Consultancy. Netherland, 2006.

BUENO, W. C. Comunicação Empresarial e Responsabilidade Social. 2006. Disponível em: <http://www.metodista.br/poscom/cientifico/publi cacoes/docentes/artigos/artigo-0050>. Acesso em: 15 mar. 2014.

DE DEAR, R. Thermal Comfort in Practice. Indoor Air Journal, v. 14, supl. 7, p. 32-39, 2004.

DIAS, A. Definição dos Parâmetros de Conforto Térmico Para os Usuários Adolescentes de Edificações Escolares. Brasília, 2009. Dissertação (Mestrado em Arquitetura e Urbanismo) - Escola de Engenharia, Universidade de Brasília, Brasília, 2009.

FANGER, P. O. Thermal Comfort. Copenhagen: Danish Technical Press, 1970. Technical University of Denmark, Laboratory of heating and Air Conditioning.

COUTINHO FILHO, E. F; SILVA, E. C. S. da; SILVA, L. B. da. Avaliação do Conforto Ambiental em Uma Escola Municipal de João Pessoa. In: ENCONTRO DE EXTENSÃO UNIVERSITÁRIA. DESAFIOS DA INDISSOCIABILIDADE ENTRE ENSINO E EXTENSÃO, 9., João Pessoa, 2007. Anais... João Pessoa: Editora Universitária da UFPB, 2007.

GRZYBOWSKI, G. T. Conforto Térmico nas Escolas Públicas em Cuiabá - MT: estudo de caso. Cuiabá, 2004. Dissertação (Mestrado em Física e Meio Ambiente) - Escola de Engenharia, Universidade Federal de Mato Grosso, Cuiabá, 2004.

HELLWIG, R. T.; BISCHOF, W. Gültigkeit Thermischer Behaglichkeitsmodelle. Bauphysik Journal, v. 28, n. 2, p. 131-136, 2006.

HUMPHREYS, M. A. Field Studies of Thermal Comfort Compared and Applied. Building Services Engineer, Watfort, v. 44, p. 5-27, 1976.

INSTITUTO BRASILEIRO DE GEOGRAFIA E ESTATÍSTICA. Censo Demográfico: Brasil. Rio de Janeiro, 2014.
INTERNATIONAL ORGANIZATION FOR STANDARDIZATION. ISO 7730: ergonomics of the thermal environment: instruments and methods for measuring physical quantities. Geneva, 2005.

\section{INTERNATIONAL ORGANIZATION FOR}

STANDARDIZATION. ISO 7726: ergonomics of the thermal environment: instruments and methods for measuring physical quantities. Geneva, 1998.

\section{KUCHEN, E. Spot-Monitoring Zum}

Thermischen Komfort in Bürogebäude. $203 \mathrm{f}$. Braunschweig, 2008. Thesis (Ph.D.) - Technische Universität Braunschweig, Braunschweig, 2008.

KUCHEN, E.; FISCH, M. N. Spot Monitoring: Thermal Comfort Evaluation in 25 Office Buildings in Winter. Building and Environment Journal, v. 44, n. 4, p. 839-847, 2009.

KUCHEN, E. et al. Predição do Índice de CONFORTO TÉRMICO em Edifícios de Escritório na Alemanha. Ambiente Construído, Porto Alegre, v. 11, n. 3, p. 39-53, jul./set. 2011.

MAITELLI, G. T. Interações AtmosferaSuperfície. In: MORENO, G.; HIGA, T. C. S. Geografia de Mato Grosso: território, sociedade, ambiente. Cuiabá: Editora Estrelinhas, 2005.

NICOL, J. F.; HUMPHREYS, M. A. Adaptive Thermal Comfort and Sustainable Thermal Standards for Buildings. Energy and Buildings Journal, v. 34, n. 6, p. 563-572, 2002.

NOGUEIRA, M. C. J. A.; NOGUEIRA, J. S. Educação, Meio Ambiente e Conforto Térmico: caminhos que se cruzam. Revista Eletrônica em Educação Ambiental. Rio Grande, v. 10, p. 104108, 2003.

OSELAND, N. A. A Comparison of the Predicted and Reported Thermal Sensation Vote in Homes During Winter and Summer. Energy and Buildings, Lausanne, v. 21, n. 1, p. 45-54, 1994.

RAUE, A. K. et al. Buildings versus HVAC Buildings: a new dutch thermal comfort guideline. In: AIVC CONFERENCE, 25., Prague, 2004. Proceedings... Prague, 2004.

SANCHES, J. C. M.; QUEIROZ, T. M.; VIEIRA, N. L. Viabilidade da utilização de Coberturas Verdes em Mato Grosso Segundo o Balanço Hídrico Climatológico do Estado e o Zoneamento Bioclimático brasileiro. In: SEMINÁRIO DE HABITAÇÃO E INTERESSE SOCIAL, 4., Sinop, 2011. Anais... Sinop, 2011.

TARIFA, J. R. Atlas de Mato Grosso: abordagem socioeconômico-ecológica. Cuiabá: Entrelinhas, 2011. 
XAVIER, A. A. P. Condições de Conforto Térmico Para Estudantes de ${ }^{\circ}$ Grau na Região de Florianópolis. Florianópolis, 1999. Dissertação (Mestrado em Engenharia Civil) - Escola de Engenharia, Universidade Federal de Santa Catarina, Florianópolis, 1999.

\section{Agradecimentos}

Os autores agradecem ao CNPq pelos equipamentos de medição que permitiram o desenvolvimento deste trabalho.

\section{Karen Wrobel Straub}

Departamento de Engenharia Civil | Universidade do Estado de Mato Grosso | Av. dos Ingás, 3001, Jardim Imperial | Sinop - MT - Brasil | CEP 78555-000 | Tel.: (66) 3511-2100 | E-mail: karen.straub@unemat.br

Erika Fernanda Toledo Borges Leão

Departamento de Engenharia Civil | Universidade do Estado de Mato Grosso | E-mail: borgesleao@unemat-net.br

\section{Ernesto Kuchen}

Instituto Regional de Planeamiento y Hábitat | Universidad Nacional de San Juan | Av. Ignacio de La Roza, 590, Oeste | Rivadavia - San Juan - Argentina | CP5400 | Tel.: (54) 264 423-3259 | E-mail: ernestokuchen@faud.unsj.edu.ar

Marlon Leão

Departamento de Engenharia Civil | Universidade do Estado de Mato Grosso | E-mail: leao@unemat.br

\section{Revista Ambiente Construído}

Associação Nacional de Tecnologia do Ambiente Construído

Av. Osvaldo Aranha, $99-3^{\circ}$ andar, Centro

Porto Alegre - RS - Brasil

CEP $90035-190$

Telefone: +55 (51) 3308-4084

Fax: +55 (51) 3308-4054

www.seer.ufrgs.br/ambienteconstruido

E-mail: ambienteconstruido@ufrgs.br 